\title{
Decoherence as an Inherent Characteristic of Quantum Mechanics
}

\author{
Riuji Mochizuki \\ Laboratory of Physics, Tokyo Dental College, Tokyo, Japan \\ Email: rjmochi@tdc.ac.jp
}

How to cite this paper: Mochizuki, R. (2021) Decoherence as an Inherent Characteristic of Quantum Mechanics. Journal of Modern Physics, 12, 700-729.

https://doi.org/10.4236/jmp.2021.125045

Received: March 19, 2021

Accepted: April 26, 2021

Published: April 29, 2021

Copyright () 2021 by author(s) and Scientific Research Publishing Inc.

This work is licensed under the Creative

Commons Attribution International

License (CC BY 4.0).

http://creativecommons.org/licenses/by/4.0/

\begin{abstract}
In this study, we show that it is possible to explain the quantum measurement process within the framework of quantum mechanics without any additional postulates. We do not delve into a deep discussion regarding what the measurement problem actually is, and only examine the problems that seem to exist between classical and quantum physics. Relations between quantum and classical equations of motion are briefly reviewed to show that the transition from a superposition of quantum states to an eigenstate, namely, decoherence, is necessary to ensure that the expectation values in quantum mechanics obey the classical equations of motion. Several Bell-type inequalities and the Kochen-Specker theorem are also reviewed to clarify the concepts of nonseparability and counterfactual definiteness in quantum mechanics. The main objective of this study is to show that decoherence is an inherent characteristic of quantum states caused by the quantum uncertainty relation. We conclude that the quantum measurement process can indeed be explained within the framework of pure quantum mechanics. We also show that our conclusion is consistent with the counterfactual indefiniteness of quantum mechanics.
\end{abstract}

\section{Keywords}

Decoherence, Uncertainty Relation, Measurement Problem

\section{Introduction}

The measurement problem in quantum mechanics is an unresolved problem in modern physics, and a subject of considerable debate [1] [2]. The microscopic world in conventional quantum mechanics is treated differently from the macroscopic world in classical mechanics. However, these two worlds are, in fact, linked to each other. Therefore, it may seem that the present formulation of quantum mechanics is insufficient to describe nature. Nevertheless, for many 
decades, it has been unclear how exactly to formulate the measurement problem, which has led to several definitions of this problem.

For a better understanding of the discussion in subsequent sections, we first provide some background on the problems that exist between classical physics and quantum mechanics, and the theories suggested to solve them. In the present work, we examine a Stern-Gerlach-like experiment, where the spin in the $z$-direction of an electron $S$ is measured, to clarify these problems.

In classical mechanics, the relation between the state of a system under investigation and the physical quantities associated with it is trivial. However, certain assumptions are needed to connect these in quantum mechanics. In its standard formulation, the eigenstate-eigenvalue link [3] [4] states that eigenvalues and eigenstates have a one-to-one correspondence except in the case of degeneracy. Thus, in our Stern-Gerlach-like experiment, we assume that

$$
\begin{aligned}
& \text { eigenvalue }+\hbar / 2 \rightleftharpoons \text { eignestate }|+\rangle, \\
& \text { eigenvalue }-\hbar / 2 \rightleftharpoons \text { eigenstate }|-\rangle,
\end{aligned}
$$

where $|+\rangle$ and $|-\rangle$ represent the eigenstates of $S$ with eigenvalues $+\hbar / 2$ and $-\hbar / 2$, respectively.

However, some studies have suggested that this condition is unnecessary [5]. Nevertheless, if we do not agree with the eigenstate-eigenvalue link, then it follows that standard quantum mechanics is insufficient to describe nature. To better understand this, we examine the above assumption in more detail. Note that because the assumption eigenstate $\rightarrow$ eigenvalue is a part of Born's rule [6], and discarding this assumption implies discarding Born's rule. Conversely, discarding the assumption eigenvalue $\rightarrow$ eigenstate implies that there may be states other than the eigenstate that correspond to the measured value of the concerned physical quantity. For instance, let $a_{i}$ be the value obtained after the measurement of a quantity. If the state obtained directly after this measurement is not the eigenstate corresponding to $a_{i}$, then it is possible to obtain a measured value that is different from $a_{i}$ even after the first measurement. Thus, if we discard the assumption eigenvalue $\rightarrow$ eigenstate, then the standard theory of quantum mechanics appears to be insufficient.

We introduce an ideal measurement device $M$ and assume that $S$ and $M$ interact by obeying the Schrödinger equation. Let $|n\rangle$ be the neutral state of $M$ before measurement, and $|p\rangle$ and $|m\rangle$ be the states of $M$ after measurement with measured values $+\hbar / 2$ and $-\hbar / 2$, respectively. Subsequently, taking into account the eigenstate-eigenvalue link, an ideal measurement process $\hat{U}$ is defined as

$$
\begin{aligned}
& |+\rangle|n\rangle \rightarrow \hat{U}|+\rangle|n\rangle=|+\rangle|p\rangle, \\
& |-\rangle|n\rangle \rightarrow \hat{U}|-\rangle|n\rangle=|-\rangle|m\rangle .
\end{aligned}
$$

Let the initial state of $S$ be $(1 / \sqrt{2})(|+\rangle+|-\rangle)$. Then, the state obtained after the measurement process is given by 


$$
\hat{U} \frac{1}{\sqrt{2}}(|+\rangle+|-\rangle)|n\rangle=\frac{1}{\sqrt{2}}(|+\rangle|p\rangle+|-\rangle|m\rangle) .
$$

Note that either the measured value $+\hbar / 2$ or $-\hbar / 2$ is expected as a result of the measurement in Equation (5). However, the measurement process and the eigenstate-eigenvalue link contradict each other because the initial state of $S$ is not the eigenstate corresponding to the eigenvalues $+\hbar / 2$ or $-\hbar / 2$.

Thus, the above example shows that it is necessary to adopt a modification of standard quantum mechanics. The most traditional approach is to adopt the projection postulate [7]. As will be discussed in Section 2, the transition from a superposition of states to an eigenstate is necessary so that the expectation values in quantum mechanics obey the classical equations of motion. However, this transition cannot be described by the Schrödinger equation because it is not a unitary process. By adopting the projection postulate, the quantum states not only develop unitarily by obeying the Schrödinger equation but also non-unitarily owing to the collapse of the wave packets during the measurement process. For example, a state $|\psi\rangle$ changes to the corresponding eigenstates $\left|a_{i}\right\rangle$ after the measurement with corresponding measured values $a_{i}$. Thus, in the Stern-Gerlach-like experiment, a non-unitary change such as

$$
\frac{1}{\sqrt{2}}(|+\rangle|p\rangle+|-\rangle|m\rangle) \rightarrow|+\rangle|p\rangle \text { or }|-\rangle|m\rangle
$$

is permitted in addition to the unitary development represented by Equation (5).

Although the projection postulate is a type of nonlocal requirement, there are several studies that demonstrate its consistency with special relativity [8]. Note that classical information cannot be transferred during the collapse of a wave packet.

In contrast to the above description, the collapse of a wave packet is not assumed in the many-worlds interpretation of quantum mechanics [9] [10]. In this interpretation, even macroscopic states maintain coherent superpositions, and therefore, the assumption that only one outcome can be obtained from one appropriate measurement process, which is usually regarded as a matter of course, is discarded.

Decoherence [11] [12] may be regarded as one of the most successful theories to explain the quantum measurement process without any additional postulates. Moreover, it is frequently applied to the many-worlds interpretation [13]. Decoherence was first proposed by Zeh et al. [14] [15], which was followed by the important work of Zurek [16]. The efforts of these authors ensured that the decoherence theory was actively studied. In this theory, the observed decay of interference is explained by the interactions between the system and the environmental degrees of freedom. For example, let the state $|Z\rangle$ that represents a unified system consisting of an observed system and a measurement device be described by the superposition of the two states $|a\rangle$ and $|b\rangle$, such that:

$$
|Z\rangle=\frac{1}{\sqrt{2}}(|a\rangle+|b\rangle)
$$


Next, we suppose that this state interacts with the state $\left|E_{0}\right\rangle$ representing the environmental degrees of freedom. The interaction dynamics between these states are given by

$$
\begin{aligned}
|a\rangle\left|E_{0}\right\rangle & \rightarrow|a\rangle\left|E_{a}\right\rangle, \\
|b\rangle\left|E_{0}\right\rangle & \rightarrow|b\rangle\left|E_{b}\right\rangle .
\end{aligned}
$$

The density matrix $\hat{\rho}_{Z}$ of the interacting system is then

$$
\hat{\rho}_{Z}=\frac{1}{2}\left(|a\rangle\left|E_{a}\right\rangle+|b\rangle\left|E_{b}\right\rangle\right)\left(\langle a|\left\langle E_{a}\right|+\langle b|\left\langle E_{b}\right|\right) .
$$

Note that because the environmental degrees of freedom are very large, the states $\left|E_{a}\right\rangle$ and $\left|E_{b}\right\rangle$ resulting from the interaction are approximately orthogonal if $|a\rangle$ and $|b\rangle$ are distinguishable. Therefore, the reduced density matrix $\hat{\rho}_{r e z}$ of the unified system, which is obtained by tracing out the environmental degrees of freedom, becomes

$$
\hat{\rho}_{r e Z}=\frac{1}{2}(|a\rangle\langle a|+| b\rangle\langle b|) .
$$

Thus, we observe the decay of interference in the system. Moreover, the preferred basis problem has also been studied in this framework by Zurek [17], who also presented a solution for this problem.

However, certain problems remain with the decoherence theory. First, the unitary interaction between the system and the environment does not lead to a complete removal of the interference terms. The reduced density matrix given by Equation (11) does not indicate whether the system is in state $|a\rangle$ or $|b\rangle$ because reduced density matrices represent improper mixtures [18] that only provide a probability distribution. This implies that the coherence has been delocalised into a larger system including the environment [2]. The next problem may be more severe. Note that because the coherent terms disappear after in the interaction between the system and the environment, obtaining an outcome for a superposition of states implies direct violation of the eigenstate-eigenvalue link.

Considering the above arguments, it seems necessary to remove certain natural conditions from or add certain new unnatural conditions to standard quantum mechanics to explain the measurement process. In other words, the quantum measurement problem is reduced to the problem of determining these conditions.

Note that in the present study, we do not delve into a discussion regarding what the measurement problem actually is. Instead, we define our problem in the form of the following question: is it possible to explain the measurement process within the framework of pure quantum mechanics? In this study, pure quantum mechanics refers to the theory of quantum mechanics without any additional postulates, such as the projection postulate. We demonstrate that the answer to this question is, in fact, yes; thus, we can indeed explain the measurement process within the framework of pure quantum mechanics.

The remaining paper is organised as follows. 
In Section 2, the relations between the quantum mechanical and classical equations of motion are briefly reviewed. It is shown that the transition from a superposition of states to an eigenstate is necessary to ensure that the expectation values in quantum mechanics obey the classical equations of motion; specifically, decoherence is necessary to connect the classical and quantum worlds.

In Section 3, several Bell-type inequalities are reviewed. The fact that these inequalities do not hold true for quantum mechanics indicates the presence of some sort of nonlocality. Although it is unlikely that the universe is nonlocal, the measurement problem of quantum mechanics is not a problem of explaining the nonlocality itself. The question we should rather ask is whether such theories of quantum mechanics are reasonable enough to explain the measurement process and experiments that demonstrate the reasonableness of its own. The Kochen-Specker theorem is also discussed in Section 3. It is well known that this theorem prohibits counterfactual definiteness of quantum mechanics. Nevertheless, it is important to consider the meaning of counterfactual definiteness in relation to the nonlocality.

Section 4 presents the main findings of this study. We show that decoherence is an inherent characteristic of quantum states caused by the quantum uncertainty relation, and it can explain the measurement process within the framework of pure quantum mechanics. It is also shown that there is no inconsistency between decoherence and the counterfactual definiteness discussed in Section 3.

Section 5 concludes the paper.

\section{Relation between Quantum Mechanical and Classical Equations of Motion}

In this section, we show that the transition from a superposition of states to an eigenstate is necessary to ensure that the quantum mechanical expectation values obey the classical equations of motion.

\subsection{Schrödinger Equation, Path Integral Formulation, and Hamilton-Jacobi Equation}

The time evolution of physical quantities characterising macroscopic objects is described by the equations of motion in classical mechanics. Conversely, the operators corresponding to the physical quantities characterising microscopic objects do not evolve with time but the states evolve according to Schrödinger equation in the Schrödinger picture of quantum mechanics. Thus, the relation between Schrödinger equation and classical equations of motions is nontrivial. However, it can be visualised easily by adopting the path integral formulation.

\subsubsection{Relation between Schrödinger Equation and Hamilton-Jacobi Equation}

It has been shown that the phase of a wave function obeys the classical Hamilton-Jacobi equation under certain assumptions [19] [20]. Let $q_{i}(t)$ be the position at time $t$ of a particle moving in a real field $U\left(q_{i}(t), t\right)$ and $\psi\left(q_{i}(t), t\right)$ 
be its wave function, where $i=1, \cdots, d . \psi\left(q_{i}(t), t\right)$ obeys the Schrödinger equation such that

$$
i \hbar \frac{\partial \psi\left(q_{i}(t), t\right)}{\partial t}=-\frac{\hbar^{2}}{2 m} \Delta \psi\left(q_{i}(t), t\right)+U\left(q_{i}(t), t\right) \psi\left(q_{i}(t), t\right) .
$$

If we separate the wave function $\psi\left(q_{i}(t), t\right)$ into its absolute value $A\left(q_{i}(t), t\right)$ and phase $S\left(q_{i}(t), t\right) / \hbar$, such that

$$
\psi\left(q_{i}(t), t\right)=A\left(q_{i}(t), t\right) \exp \left(\frac{i}{\hbar} S\left(q_{i}(t), t\right)\right),
$$

then Equation (12) can be separated into its real and imaginary parts. The real part is given by

$$
-A \frac{\partial S}{\partial t}=-\frac{1}{2 m}\left(\hbar^{2} \Delta A-A(\nabla S)^{2}\right)+U A,
$$

and the imaginary part is given by

$$
\frac{\partial A}{\partial t}=-\frac{1}{2 m}(2 \nabla A \cdot \nabla S+A \Delta S) .
$$

Let us first consider the imaginary part in Equation (15). The right-hand side of Equation (15) multiplied by $A$ yields

$$
-\frac{A}{2 m}(2 \nabla A \cdot \nabla S+A \Delta S)=-\frac{1}{2 m} \nabla \cdot\left(A^{2} \nabla S\right) .
$$

Assuming that the momentum $p$ of the particle is $p_{i}=\nabla_{i} S$, then Equation (15) multiplied by $A$ results in the continuity equation

$$
\frac{\partial \rho}{\partial t}=-\nabla \cdot(\rho v)
$$

where $v=p / m$ is the velocity of the particle and $\rho \equiv A^{2}$.

Furthermore, ignoring the terms of $O\left(\hbar^{2}\right)$, Equation (14) becomes

$$
\frac{\partial S}{\partial t}+\frac{1}{2 m}(\nabla S)^{2}+U=0 .
$$

This is the classical Hamilton-Jacobi equation, assuming that $S$ is the action and $\nabla S$ is the momentum of the particle.

Reasonableness of these assumptions based on the similarity between quantum mechanics and optics has been discussed in several textbooks [21] [22].

\subsubsection{Path Integral Quantisation}

Note that in this section, we omit the subscript $i$ in $q_{i}$ for simplicity. Adopting the path integral quantisation method [23] makes the discussion in the previous section clearer. In this theory, the wave function $\psi\left(q_{F} \equiv q\left(t_{F}\right), t_{F}\right)$ at time $t_{F}$ can be described as evolving from the wave function $\psi\left(q_{I} \equiv q\left(t_{I}\right), t_{I}\right)$ at time $t_{I},\left(t_{I}<t_{F}\right)$, such that

$$
\psi\left(q_{F}, t_{F}\right)=\int \mathcal{D} q \exp \left(\frac{i}{\hbar} \int_{t_{I}}^{t_{F}} L(q, \dot{q}) \mathrm{d} t\right) \psi\left(q_{I}, t_{I}\right),
$$

where $L(q, \dot{q})$ is the Lagrangian and 


$$
\mathcal{D} q \equiv \lim _{n \rightarrow \infty} \prod_{r=0}^{n} \mathrm{~d} q_{r}, q_{r} \equiv q\left(t_{r}\right),
$$

with $q_{n+1}=q_{F}, q_{0}=q_{I}$. Differentiating $\psi\left(q_{F}, t_{F}\right)$ with respect to $t_{F}$, we obtain

$$
\frac{\partial \psi\left(q_{F}, t_{F}\right)}{\partial t_{F}}=\int \mathcal{D} q \frac{i}{\hbar} \frac{\partial S}{\partial t_{F}} \exp \left(\frac{i}{\hbar} \int_{t_{I}}^{t_{F}} L(q, \dot{q}) \mathrm{d} t\right) \psi\left(q_{I}, t_{I}\right)
$$

where

$$
S \equiv \int_{t_{I}}^{t_{F}} L(q, \dot{q}) \mathrm{d} t
$$

On the other hand, applying the Hamiltonian operator $\hat{H}\left(\hat{q}_{F}, \hat{p}_{F}\right)$, where $\hat{p}_{F} \equiv-i \hbar\left(\partial / \partial q_{F}\right)$, on the wave function $\psi\left(q_{F}, t_{F}\right)$ in Equation (19), we obtain

$$
\hat{H}\left(\hat{q}_{F}, \hat{p}_{F}\right) \psi\left(q_{F}, t_{F}\right)=\int \mathcal{D} q H\left(q_{F}, \partial S / \partial q_{F}\right) \exp \left(\frac{i}{\hbar} \int_{t_{I}}^{t_{F}} L(q, \dot{q}) \mathrm{d} t\right) \psi\left(q_{I}: t_{I}\right),
$$

where the terms of $\mathcal{O}(\hbar)$ or higher are ignored. We can see that $H\left(q_{F}, \partial S / \partial q_{F}\right)$ on the right-hand side of Equation (23) is the classical Hamiltonian function with $p_{F}$ substituted by $\partial S / \partial q_{F}$ on comparing Equation (23) with

$$
\begin{aligned}
\frac{\partial \psi\left(q_{F}, t_{F}\right)}{\partial t_{F}} & =\frac{\mathrm{d} \psi\left(q_{F}, t_{F}\right)}{\mathrm{d} t_{F}}-\frac{\partial \psi\left(q_{F}, t_{F}\right)}{\partial q_{F}} \dot{q}_{F} \\
& =\frac{i}{\hbar} \int \mathcal{D} q\left(L\left(q_{F}, \dot{q}_{F}\right)-\frac{\partial S}{\partial q_{F}} \dot{q}_{F}\right) \exp \left(\frac{i}{\hbar} \int_{t_{I}}^{t_{F}} L(q, \dot{q}) \mathrm{d} t\right) \psi\left(q_{I}, t_{I}\right)
\end{aligned}
$$

and the Schrödinger equation

$$
i \hbar \frac{\partial \psi\left(q_{F}, t_{F}\right)}{\partial t_{F}}=\hat{H}\left(\hat{q}_{F}, \hat{p}_{F}\right) \psi\left(q_{F}, t_{F}\right) .
$$

Substituting Equations (21) and (23) into Equation (25), we obtain the Hamilton-Jacobi equation

$$
\frac{\partial S}{\partial t_{F}}+H\left(q_{F}, \partial S / \partial q_{F}\right)=0
$$

Then, classical mechanics states that $q_{F}$ and $p_{F}=\partial S / \partial q_{F}$ must satisfy Hamilton's equations of motion:

$$
\begin{gathered}
\dot{q}_{F}-\frac{\partial H}{\partial p_{F}}=0, \\
\dot{p}_{F}+\frac{\partial H}{\partial q_{F}}=0 .
\end{gathered}
$$

As shown above, the classical equations of motion can be derived from the Schrödinger equation in the limit $\hbar \rightarrow 0$ without any analogy, if the path integral quantisation method is applied to the wave function. However, it is worth noting that the derived classical equations of motion contain no information about the initial state of the system because the information $\psi\left(q_{I}, t_{I}\right)$ has been lost in the above formalism; specifically, the discussion here is formal and 
hence does not reflect the state of the system. So far, all we have shown with the aid of quantum mechanics is that classical quantities obey classical equations of motion. Therefore, we still need to investigate the equations that the corresponding quantities in quantum mechanics (i.e. the expectation values of an operator) obey in the classical limit.

\subsection{Ehrenfest Theorem}

The Ehrenfest theorem [24] demonstrates how quantum mechanical expectation values obey the classical equations of motion. Although Ehrenfest [25] originally proposed the theorem in the Schrödinger picture, in this section we employ the Heisenberg picture. Note that because the operators corresponding to the physical quantities depend explicitly on time in the Heisenberg picture, the relation is rather straightforward. Let $\langle\hat{q}\rangle$ and $\langle\hat{p}\rangle$ be the expectation values of the coordinate $\hat{q}$ and momentum $\hat{p}$, respectively; then, the equations obeyed by $\langle\hat{q}\rangle$ and $\langle\hat{p}\rangle$ in quantum mechanics should be the same as the classical Hamilton's equations of motion under certain conditions.

Let us suppose that a quantum mechanical system is described by coordinates $\hat{q}_{i}(i=1, \cdots, N)$ and their respective conjugate momenta $\hat{p}_{i}(i=1, \cdots, N)$. Operator $\hat{A}$, which does not depend on time in the Schrödinger picture, evolves in the Heisenberg picture obeying

$$
\frac{\mathrm{d} \hat{A}}{\mathrm{~d} t}=\frac{1}{i \hbar}[\hat{A}, \hat{H}],
$$

where $\hat{H}$ is the Hamiltonian operator and [,] represents a commutator. Because the commutator between $\hat{q}_{i}$ and $\hat{p}_{j}$ is

$$
\left[\hat{q}_{i}, \hat{p}_{j}\right]=i \hbar \delta_{i j} \text {, }
$$

therefore, $\hat{H}$ satisfies

$$
\begin{gathered}
{\left[\hat{q}_{i}, \hat{H}\right]=i \hbar \frac{\partial \hat{H}}{\partial \hat{p}},} \\
{\left[\hat{p}_{i}, \hat{H}\right]=-i \hbar \frac{\partial \hat{H}}{\partial \hat{q}} .}
\end{gathered}
$$

Next, substituting $\hat{A}$ with $\hat{q}_{i}$ and $\hat{p}_{i}$ in Equation (29) and using Equations (31) and (32), we obtain the evolution equations for the respective expectation values as

$$
\begin{gathered}
\frac{\mathrm{d}}{\mathrm{d} t}\left\langle\hat{q}_{i}\right\rangle=\left\langle\frac{\partial \hat{H}}{\partial \hat{p}_{i}}\right\rangle, \\
\frac{\mathrm{d}}{\mathrm{d} t}\left\langle\hat{p}_{i}\right\rangle=-\left\langle\frac{\partial \hat{H}}{\partial \hat{q}_{i}}\right\rangle .
\end{gathered}
$$

Furthermore, if we assume

$$
\left\langle\frac{\partial \hat{H}(\hat{q}, \hat{p})}{\partial \hat{q}_{i}}\right\rangle=\frac{\partial H(\langle q\rangle,\langle p\rangle)}{\partial\left\langle q_{i}\right\rangle},
$$




$$
\left\langle\frac{\partial \hat{H}(\hat{q}, \hat{p})}{\partial \hat{p}_{i}}\right\rangle=\frac{\partial H(\langle q\rangle,\langle p\rangle)}{\partial\left\langle p_{i}\right\rangle},
$$

where $H$ is the classical Hamiltonian, we obtain

$$
\begin{gathered}
\frac{\mathrm{d}}{\mathrm{d} t}\left\langle\hat{q}_{i}\right\rangle=\frac{\partial H(\langle q\rangle,\langle p\rangle)}{\partial\left\langle p_{i}\right\rangle}, \\
\frac{\mathrm{d}}{\mathrm{d} t}\left\langle\hat{p}_{i}\right\rangle=-\frac{\partial H(\langle q\rangle,\langle p\rangle)}{\partial\left\langle q_{i}\right\rangle} .
\end{gathered}
$$

We note that the above equations are in the form of Hamilton's equations of motion in classical mechanics.

Note that the assumptions in Equations (35) and (36) are justified if $\hat{H}$ is quadratic in $\hat{q}$ and $\hat{p}$ or if the dispersion of the measured $\hat{q}$ and $\hat{p}$ values are small. The dispersion is expected to be small for eigenstates and large for a superposition of states. Therefore, the superposition of states must evolve into eigenstates to ensure that the expectation values of quantum mechanical quantities obey the classical equations of motion.

\section{Bell-Type Inequalities and the Kochen-Specker Theorem}

In this section, we review Bell-type inequalities and the Kochen-Specker theorem. These theorems indicate that the world described by quantum mechanics is considerably different from the macroscopic world we are familiar with.

It has been experimentally confirmed [26]-[32] that quantum mechanical expectation values violate Bell-type inequalities, strongly suggesting that the quantum mechanical world is nonlocal or nonseparable. D'Espagnat [18] defined separability as follows: If a physical system remains, during a certain time, mechanically (including electromagnetically, etc.) isolated from other systems, then the evolution of its properties during this whole time interval cannot be influenced by operations carried out on other systems. Conversely, the Kochen-Specker theorem implies that quantum mechanics is counterfactually indefinite. Nevertheless, the meaning of counterfactual definiteness should be considered in relation to the nonlocality of quantum mechanics; specifically, the Kochen-Specker theorem forbids quantum mechanics to be locally counterfactually definite. In other words, quantum mechanics is not forbidden to be nonlocally counterfactually definite by Bell-type inequalities or the Kochen-Specker theorem. This fact is important to the consisitency of discussion in Subsection 4.2.

\subsection{EPR-Bohm Experiment}

First, we introduce the Einstein-Podolsky-Rosen (EPR)-Bohm experiment [33] [34], in which the spins of two spin 1/2 particles, labelled as 1 and 2 in Figure 1, are measured. The sum of their spins should be zero, and their initial state $|C\rangle$ can be written as 


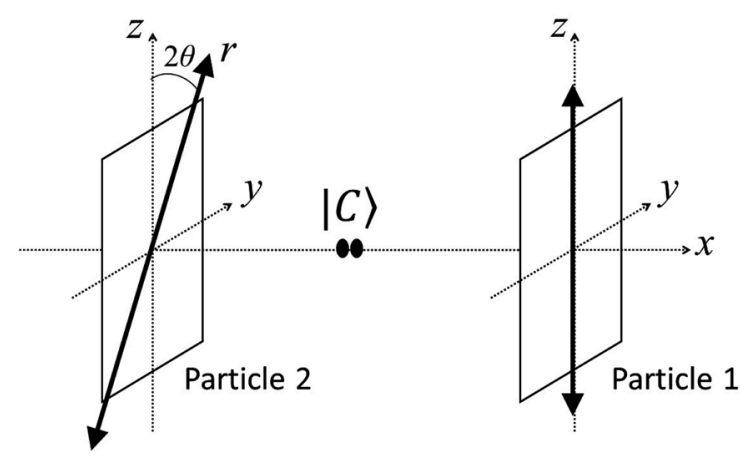

Figure 1. EPR-Bohm experiment.

$$
|C\rangle=\frac{1}{\sqrt{2}}\left(|u\rangle_{1}|d\rangle_{2}-|d\rangle_{1}|u\rangle_{2}\right),
$$

where $|u\rangle_{i}$ and $|d\rangle_{i} \quad(i=1,2)$ are the spin eigenstates in the $z$ direction. These satisfy

$$
\begin{aligned}
\left(\hat{\sigma}_{z}\right)_{i}|u\rangle_{i} & =+\frac{\hbar}{2}|u\rangle_{i}, \\
\left(\hat{\sigma}_{z}\right)_{i}|d\rangle_{i} & =-\frac{\hbar}{2}|d\rangle_{i},
\end{aligned}
$$

where $\hat{\sigma}_{z}$ is the spin operator in the $z$ direction. The spin operator in the $r$ direction, which is perpendicular to the direction of the particles' motion (i.e. along $x$ direction) and makes an angle $2 \theta$ with the $z$ direction in the $y z$-plane, is given by

$$
\hat{\sigma}_{r}=\left(\begin{array}{cc}
\cos \theta & -\sin \theta \\
\sin \theta & \cos \theta
\end{array}\right) \hat{\sigma}_{z}\left(\begin{array}{cc}
\cos \theta & \sin \theta \\
-\sin \theta & \cos \theta
\end{array}\right) .
$$

The eigenstates of $\hat{\sigma}_{r}$ are $|+\rangle_{i}$ and $|-\rangle_{i} \quad(i=1,2)$, given by

$$
\begin{aligned}
& |+\rangle_{i}=\cos \theta|u\rangle_{i}+\sin \theta|d\rangle_{i}, \\
& |-\rangle_{i}=-\sin \theta|u\rangle_{i}+\cos \theta|d\rangle_{i},
\end{aligned}
$$

which satisfy the following equations:

$$
\begin{gathered}
\left(\hat{\sigma}_{r}\right)_{i}|+\rangle_{i}=+\frac{\hbar}{2}|+\rangle_{i}, \\
\left(\hat{\sigma}_{r}\right)_{i}|-\rangle_{i}=-\frac{\hbar}{2}|-\rangle_{i} .
\end{gathered}
$$

By using Equation (43), $|C\rangle$ can be rewritten as

$$
|C\rangle=\frac{1}{\sqrt{2}}\left(|+\rangle_{1}|-\rangle_{2}-|-\rangle_{1}|+\rangle_{2}\right) .
$$

Let us suppose that two observers simultaneously perform measurements on the spins of particles 1 and 2 along the directions $z$ and $r$, respectively. Rewriting $|C\rangle$ as

$$
|C\rangle=\frac{1}{\sqrt{2}}\left[|u\rangle_{1}\left(\sin \theta|+\rangle_{2}+\cos \theta|-\rangle_{2}\right)-|d\rangle_{1}\left(\cos \theta|+\rangle_{2}-\sin \theta|-\rangle_{2}\right)\right],
$$


we can easily calculate the probabilities of observing the spin combinations $\left(+\frac{\hbar}{2},+\frac{\hbar}{2}\right),\left(+\frac{\hbar}{2},-\frac{\hbar}{2}\right),\left(-\frac{\hbar}{2},+\frac{\hbar}{2}\right)$, and $\left(-\frac{\hbar}{2},-\frac{\hbar}{2}\right)$, which are given by

$$
\begin{gathered}
P_{(+,+)} \equiv\left\langle C\left|(|u\rangle\langle u|)_{1}(|+\rangle\langle+|)_{2}\right| C\right\rangle=\frac{1}{2} \sin ^{2} \theta, \\
P_{(+,-)} \equiv\left\langle C\left|(|u\rangle\langle u|)_{1}(|-\rangle\langle-|)_{2}\right| C\right\rangle=\frac{1}{2} \cos ^{2} \theta, \\
P_{(-,+)} \equiv\left\langle C\left|(|d\rangle\langle d|)_{1}(|+\rangle\langle+|)_{2}\right| C\right\rangle=\frac{1}{2} \cos ^{2} \theta, \\
P_{(-,-)} \equiv\left\langle C\left|(|d\rangle\langle d|)_{1}(|-\rangle\langle-|)_{2}\right| C\right\rangle=\frac{1}{2} \sin ^{2} \theta,
\end{gathered}
$$

respectively.

It should be noted that these equations hold true for the measurements in which the angle between the spins of the two particles is $2 \theta$.

\subsection{Bell's Inequality and CHSH Inequality}

\subsubsection{Derivation}

We examine the EPR-Bohm experiment in which the spin of particle 1 along directions $a$ or $a^{\prime}$ and the spin of particle 2 along directions $b$ or $b^{\prime}$ are measured. Let $A, A, B$, and $B^{\prime}$ be the measured values of the spin divided by $\hbar / 2$, respectively. In this section, we assume separability: the measurement of the spin of particle 1 never affects the measurement of the spin of particle 2 and vice versa. We also assume counterfactual definiteness: the spin value is determined separately before the measurement. In other words, each spin is assumed to have a definite value even if the measurement has not actually been performed. Thus, $A, A$, $B$, and $B$ 'assume a definite value +1 or -1 each.

Based on these assumptions, we define the quantity $M$ as

$$
M \equiv A B-A B^{\prime}+A^{\prime} B+A^{\prime} B^{\prime}=A\left(B-B^{\prime}\right)+A^{\prime}\left(B+B^{\prime}\right) .
$$

Note that because one of the terms on the right-hand side of Equation (52) is always 0 and the other term is always +2 or $-2, M$ is always +2 or -2 . Therefore, the average value $\bar{M}$ obtained after several measurements of $M$ satisfies

$$
-2 \leq \bar{M} \leq+2 .
$$

This is the CHSH inequality [35].

Next, we examine the case in which $A, A, B$, and $B$ ' are probabilistically determined by hidden local variables [2] [18] [36]. Let us suppose that the measured values depend on a set of hidden local variables represented by $\lambda$. Then, $A$, $A, B$, and $B^{\prime}$ depend on $\lambda$ and assume a value of either +1 or -1 each. Let $P_{+(-)}^{A}(\lambda)$ be the probability that $A(\lambda)$ has a value $+1(-1)$ for a given $\lambda$. The average value $\bar{A}(\lambda)$ of $A(\lambda)$ is then given by

$$
\bar{A}(\lambda)=P_{+}^{A}(\lambda)-P_{-}^{A}(\lambda) \text {. }
$$

Thus, $\bar{A}(\lambda)$ satisfies

$$
-1 \leq \bar{A}(\lambda) \leq+1
$$


Similar inequalities are satisfied by the average values of $A^{\prime}, B$, and $B^{\prime}$. The average value $\overline{A B}$ of $A B$ can be written as

$$
\begin{aligned}
\overline{A B}= & \int \mathrm{d} \lambda \rho(\lambda)\left[P_{+}^{A}(\lambda) P_{+}^{B}(\lambda)+P_{-}^{A}(\lambda) P_{-}^{B}(\lambda)\right. \\
& \left.-P_{+}^{A}(\lambda) P_{-}^{B}(\lambda)-P_{-}^{A}(\lambda) P_{+}^{B}(\lambda)\right] \\
= & \int \mathrm{d} \lambda \rho(\lambda) \bar{A}(\lambda) \bar{B}(\lambda),
\end{aligned}
$$

where $\rho(\lambda)$ is the probability distribution of $\lambda$, and $\overline{A B}$ satisfies

$$
-1 \leq \overline{A B} \leq+1 .
$$

Again, similar inequalities are satisfied by the average values of $A B^{\prime}, A^{\prime} B$ and $A^{\prime} B^{\prime}$.

Next, let us consider $\overline{A B}-\overline{A B^{\prime}}$ :

$$
\begin{aligned}
\overline{A B}-\overline{A B^{\prime}}= & \int \mathrm{d} \lambda \rho(\lambda)\left[\bar{A}(\lambda) \bar{B}(\lambda)-\bar{A}(\lambda) \overline{B^{\prime}}(\lambda)\right] \\
= & \int \mathrm{d} \lambda \rho(\lambda)\left[\bar{A}(\lambda) \bar{B}(\lambda) \pm \bar{A}(\lambda) \bar{B}(\lambda) \overline{A^{\prime}}(\lambda) \overline{B^{\prime}}(\lambda)\right. \\
& \left.\mp \bar{A}(\lambda) \bar{B}(\lambda) \overline{A^{\prime}}(\lambda) \overline{B^{\prime}}(\lambda)-\bar{A}(\lambda) \overline{B^{\prime}}(\lambda)\right] \\
= & \int \mathrm{d} \lambda \rho(\lambda) \bar{A}(\lambda) \bar{B}(\lambda)\left[1 \pm \overline{A^{\prime}}(\lambda) \overline{B^{\prime}}(\lambda)\right] \\
& -\int \mathrm{d} \lambda \rho(\lambda) \bar{A}(\lambda) \overline{B^{\prime}}(\lambda)\left[1 \pm \overline{A^{\prime}}(\lambda) \bar{B}(\lambda)\right] .
\end{aligned}
$$

From inequality (57), it follows that the absolute value of Equation (58) satisfies

$$
\left|\overline{A B}-\overline{A B^{\prime}}\right| \leq \int \mathrm{d} \lambda \rho(\lambda)\left\{\left[1 \pm \overline{A^{\prime}}(\lambda) \overline{B^{\prime}}(\lambda)\right]+\left[1 \pm \overline{A^{\prime}}(\lambda) \bar{B}(\lambda)\right]\right\}
$$

or

$$
\left|\overline{A B}-\overline{A B^{\prime}}\right| \leq 2 \pm\left[\overline{A^{\prime} B^{\prime}}+\overline{A^{\prime} B}\right] .
$$

Note that the fact that the sum of the spins of particles 1 and 2 is zero when $a=b$ has not been used in the derivation of inequality (60). If we take this into account and put $b^{\prime}=a^{\prime}$, then we can substitute $\overline{A^{\prime} B^{\prime}}=-1$ into inequality (60) to obtain

$$
\left|\overline{A B}-\overline{A B^{\prime}}\right| \leq 1+\overline{A^{\prime} B}
$$

This was the first inequality proposed by Bell in 1964 [37].

\subsubsection{Contradiction between CHSH Inequality and Quantum Mechanics} In this section, we show that quantum-mechanical expectation values violate the CHSH inequality (53).

We calculate the quantum-mechanical expectation value $\langle M\rangle$ of $M$ defined in Equation (52) using Equations (48) to (51). Let $a=z$ and $\alpha, \beta$, and $\gamma$ be the angles that directions $a^{\prime}, b$, and $b^{\prime}$ make with the $z$ direction in the $y z$-plane, respectively. The expectation value of the first term of $M$ then becomes

$$
\begin{aligned}
\langle A B\rangle & =P_{(+,+)}+P_{(-,-)}-P_{(+,-)}-P_{(-,+)} \\
& =\sin ^{2}(\beta / 2)-\cos ^{2}(\beta / 2) \\
& =-\cos \beta .
\end{aligned}
$$


The expectation values of the remaining three terms of $M$ can be similarly calculated. Thus, $\langle M\rangle$ can be written as

$$
\langle M\rangle=-\cos \beta+\cos \gamma-\cos (\beta-\alpha)-\cos (\gamma-\alpha) \text {. }
$$

We calculate the maximum and minimum values of $\langle M\rangle$ using Equation (63) to check whether it satisfies the CHSH inequality. $\langle M\rangle$ can be extremised with respect to $\alpha, \beta$, and $\gamma$, thus yielding three critical conditions. Differentiating equation (63) with respect to $\alpha$, we obtain

$$
0=\frac{\partial\langle M\rangle}{\partial \alpha}=-\sin (\beta-\alpha)-\sin (\gamma-\alpha),
$$

which yields

$$
\beta+\gamma=2 \alpha
$$

or

$$
\beta-\gamma=\pi
$$

Differentiating with respect to $\beta$, we obtain

$$
0=\frac{\partial\langle M\rangle}{\partial \beta}=\sin \beta+\sin (\beta-\alpha),
$$

which yields

$$
2 \beta=\alpha
$$

or

$$
\alpha=\pi \text {. }
$$

Finally, differentiating with respect to $\gamma$, we obtain

$$
0=\frac{\partial\langle M\rangle}{\partial \gamma}=-\sin \gamma+\sin (\gamma-\alpha),
$$

which yields

$$
\alpha=0
$$

or

$$
2 \gamma-\alpha=\pi
$$

$\langle M\rangle$, as per Equation (63), has a maximum or minimum value when Equations (65), (68), and (72) are satisfied, such that

$$
\begin{gathered}
\langle M\rangle=-2 \sqrt{2} \quad \text { when }(\alpha, \beta, \gamma)=(\pi / 2, \pi / 4,3 \pi / 4), \\
\langle M\rangle=+2 \sqrt{2} \quad \text { when }(\alpha, \beta, \gamma)=(\pi / 2,-3 \pi / 4,-\pi / 4) .
\end{gathered}
$$

Therefore, we can conclude that quantum-mechanical expectation values violate the $\mathrm{CHSH}$ inequality (53).

\subsection{Wigner's Inequality and Its Variations}

Note that we again assume counterfactual definiteness and nonseparability in the derivation of the inequalities in this section. 


\subsubsection{Wigner's Inequality}

We re-examine the EPR-Bohm type experiment introduced in Section 3.1, where the spin was measured along two of the three directions: $a, a^{\prime}$ and $a^{\prime \prime}$. Let $A$ and $B$ be the definite values of the spin along a divided by $\hbar / 2$ of particles 1 and 2, respectively. Similarly, $A^{\prime}$ and $B^{\prime}$ are the definite values along $a^{\prime}$, and $A^{\prime \prime}$ and $B^{\prime \prime}$ the definite values along $a^{\prime \prime}$. Let $\alpha$ be the angle between a and $a^{\prime}, \beta$ between $a^{\prime}$ and $a^{\prime \prime}$, and $\gamma$ between $a^{\prime \prime}$ and a. Moreover, let $P\left(A=+1, B^{\prime}=+1\right)$ be the probability that $A=+1$ and $B^{\prime}=+1$, and other probabilities can be defined similarly.

Note that because the sum of the spins of particles 1 and 2 along the same direction is zero, $P\left(A=+1, B^{\prime}=+1\right)$ can be written as

$$
\begin{aligned}
& P\left(A=+1, B^{\prime}=+1\right) \\
& =p\left(A=+1, A^{\prime}=-1, B=-1, B^{\prime}=+1\right) \\
& =p\left(A=+1, A^{\prime}=-1, A^{\prime \prime}=+1, B=-1, B^{\prime}=+1, B^{\prime \prime}=-1\right) \\
& +p\left(A=+1, A^{\prime}=-1, A^{\prime \prime}=-1, B=-1, B^{\prime}=+1, B^{\prime \prime}=+1\right),
\end{aligned}
$$

where $p\left(A=+1, A^{\prime}=-1, B=-1, B^{\prime}=+1\right)$ is the probability that the definite values are $A=+1, A^{\prime}=-1, B=-1$, and $B^{\prime}=+1$, irrespective of whether they are measurable. Similarly,

$$
\begin{aligned}
& P\left(A=+1, B^{\prime \prime}=+1\right) \\
= & p\left(A=+1, A^{\prime \prime}=-1, B=-1, B^{\prime \prime}=+1\right) \\
= & p\left(A=+1, A^{\prime}=+1, A^{\prime \prime}=-1, B=-1, B^{\prime}=-1, B^{\prime \prime}=+1\right) \\
& +p\left(A=+1, A^{\prime}=-1, A^{\prime \prime}=-1, B=-1, B^{\prime}=+1, B^{\prime \prime}=+1\right),
\end{aligned}
$$

and

$$
\begin{aligned}
& P\left(A^{\prime \prime}=+1, B^{\prime}=+1\right) \\
& =p\left(A^{\prime}=-1, A^{\prime \prime}=+1, B^{\prime}=+1, B^{\prime \prime}=-1\right) \\
& =p\left(A=+1, A^{\prime}=-1, A^{\prime \prime}=+1, B=-1, B^{\prime}=+1, B^{\prime \prime}=-1\right) \\
& +p\left(A=-1, A^{\prime}=-1, A^{\prime \prime}=+1, B=+1, B^{\prime}=+1, B^{\prime \prime}=-1\right) .
\end{aligned}
$$

Using these equations, we obtain

$$
\begin{aligned}
& P\left(A=+1, B^{\prime \prime}=+1\right)+P\left(A^{\prime \prime}=+1, B^{\prime}=+1\right) \\
& =p\left(A=+1, A^{\prime}=+1, A^{\prime \prime}=-1, B=-1, B^{\prime}=-1, B^{\prime \prime}=+1\right) \\
& +p\left(A=+1, A^{\prime}=-1, A^{\prime \prime}=-1, B=-1, B^{\prime}=+1, B^{\prime \prime}=+1\right) \\
& +p\left(A=+1, A^{\prime}=-1, A^{\prime \prime}=+1, B=-1, B^{\prime}=+1, B^{\prime \prime}=-1\right) \\
& +p\left(A=-1, A^{\prime}=-1, A^{\prime \prime}=+1, B=+1, B^{\prime}=+1, B^{\prime \prime}=-1\right) \\
& =P\left(A=+1, B^{\prime}=+1\right) \\
& +p\left(A=+1, A^{\prime}=+1, A^{\prime \prime}=-1, B=-1, B^{\prime}=-1, B^{\prime \prime}=+1\right) \\
& +p\left(A=-1, A^{\prime}=-1, A^{\prime \prime}=+1, B=+1, B^{\prime}=+1, B^{\prime \prime}=-1\right) .
\end{aligned}
$$

Recalling that probability cannot be less than zero, we obtain Wigner's inequality [38], such that

$$
P\left(A=+1, B^{\prime \prime}=+1\right)+P\left(A^{\prime \prime}=+1, B^{\prime}=+1\right) \geq P\left(A=+1, B^{\prime}=+1\right) .
$$


Using Equation (48) to confirm that the quantum-mechanical expectation value violates Wigner's inequality (79), we obtain

$$
\sin ^{2} \frac{\gamma}{2}+\sin ^{2} \frac{\beta}{2} \geq \sin ^{2} \frac{\alpha}{2}
$$

When $\alpha=2 \beta=2 \gamma$, inequality (80) becomes

$$
2 \sin ^{2} \frac{\beta}{2} \geq \sin ^{2} \beta \text {. }
$$

Moreover, if we use

$$
\sin ^{2} \beta=4 \sin ^{2} \frac{\beta}{2} \cos ^{2} \frac{\beta}{2},
$$

inequality (81) can be rewritten as

$$
\sin ^{2} \frac{\beta}{2} \geq 2 \sin ^{2} \frac{\beta}{2} \cos ^{2} \frac{\beta}{2} .
$$

The above inequality is violated when

$$
-\frac{\pi}{2}<\beta<\frac{\pi}{2} \text { except } \beta=0 .
$$

\subsubsection{Mermin's Inequality}

Mermin [39] suggested a variation of Wigner's inequality under the same conditions.

Mermin computed the probability that the spins of particles 1 and 2 measured in different directions have different signs. Let us consider separately the cases where 1) all of them have the same sign, that is, $A=A^{\prime}=A^{\prime \prime}= \pm 1$ or 2) two of them have the same sign and the other has a different sign. For example, $A=+1, A^{\prime}=A^{\prime \prime}=-1$.

In case 1), it can be shown trivially that the probability of the spins of the two particles being different is 1 . In case 2), the corresponding probability is $1 / 3$. Therefore, the probability that the spins of particles 1 and 2 measured in different directions have different signs exists between 1 and $1 / 3$. This can be expressed as

$$
1 \geq P(+1,-1)+P(-1,+1) \geq \frac{1}{3} .
$$

However, when all the angles between $a, a^{\prime}$ and $a^{\prime \prime}$ are $2 \pi / 3$, then $P(+1,-1)+P(-1,+1)$ can be calculated using Equations (49) and (50), and becomes

$$
P(+1,-1)+P(-1,+1)=\frac{1}{8}+\frac{1}{8}=\frac{1}{4},
$$

which shows that quantum mechanical probabilities violate Mermin's inequality.

\subsubsection{Albert's Inequality}

Albert [40] [41] also derived an inequality under the same conditions as Wigner's inequality. 
Note that the number of combinations of $\left(A, A^{\prime}, A^{\prime \prime} ; B, B^{\prime}, B^{\prime \prime}\right)$ is 8 considering that $A=-B, A^{\prime}=-B^{\prime}$ and $A^{\prime \prime}=-B^{\prime \prime}$. These are

$$
\begin{aligned}
& (+1,+1,+1 ;-1,-1,-1) \\
& (+1,+1,-1 ;-1,-1,+1) \\
& (+1,-1,+1 ;-1,+1,-1) \\
& (-1,+1,+1 ;+1,-1,-1) \\
& (+1,-1,-1 ;-1,+1,+1) \\
& (-1,+1,-1 ;+1,-1,+1) \\
& (-1,-1,+1 ;+1,+1,-1) \\
& (-1,-1,-1 ;+1,+1,+1)
\end{aligned}
$$

As can be easily checked, each of the above combinations satisfies at least one of the following three conditions: $A=-B^{\prime}, A^{\prime}=-B^{\prime \prime}$ and $A^{\prime \prime}=-B$. Therefore, the sum of the probabilities that one of these three conditions is satisfied is 1 or more. In other words, we can write

$$
\begin{aligned}
1 \leq & P\left(A=+1, B^{\prime}=-1\right)+P\left(A^{\prime}=+1, B^{\prime \prime}=-1\right)+P\left(A^{\prime \prime}=+1, B=-1\right) \\
& +P\left(A=-1, B^{\prime}=+1\right)+P\left(A^{\prime}=-1, B^{\prime \prime}=+1\right)+P\left(A^{\prime \prime}=-1, B=+1\right),
\end{aligned}
$$

where at least one term on the right-hand side must be $1 / 6$ or more. However, when all the angles between $a, a^{\prime}$ and $a^{\prime \prime}$ are $2 \pi / 3$, every term on the right-hand side of condition (88) is calculated to be 1/8 using Equations (49) and (50). This again shows that quantum mechanical expectation values violate Albert's inequality (88).

\subsection{Kochen-Specker Theorem}

In the previous section, we derived a series of Bell-type inequalities assuming not only separability but also counterfactual definiteness. Because quantum mechanical expectation values violate these inequalities, it implies that quantum mechanics must be non-separable or counterfactually indefinite or both. Interestingly, the Kochen-Specker theorem [42] solely requires the assumption of counterfactual definiteness.

First, we define the function $f(\hat{A})$ of an operator $\hat{A}$ as follows: $\hat{A}$ is expanded using its eigenvalues $a_{i}$ and the projection operators $\hat{P}_{i}^{A}$ on the respective states corresponding to $a_{i}$ such that

$$
\hat{A}=\sum_{i} a_{i} \hat{P}_{i}^{A} \text {. }
$$

Then, $f(\hat{A})$ can be defined as

$$
f(\hat{A}) \equiv \sum_{i} f\left(a_{i}\right) \hat{P}_{i}^{A}
$$

Let us suppose that every physical quantity $A$ has a definite value ${ }^{1}[A]$ belonging to the spectrum of its corresponding operator $\hat{A}$. In this section, we introduce an assumption that we call "FUNC", which plays an important role in ${ }^{1}$ Note that in this subsection, definite values are denoted with [ ]. 
the derivation of the Kochen-Specker theorem. According to FUNC, if an operator $\hat{B}$ is defined as $\hat{B}=f(\hat{A})$, then the definite values $[A]$ and $[B]$ satisfy $[B]=f([A])$.

Using FUNC, we derive a sum rule for the commuting operators $\hat{A}$ and $\hat{B}$ in an $N$-dimensional Hilbert space. We also define an operator $\hat{C}$, whose eigenvectors include all the vectors that form an orthonormal basis by simultaneously diagonalising $\hat{A}$ and $\hat{B} . \hat{C}$ can be expanded as

$$
\hat{C}=\sum_{i=1}^{N} c_{i} \hat{P}_{i}^{C},
$$

where $c_{i}$ represents the eigenvalues of $\hat{C}$ and $\hat{P}_{i}^{C}$ represents the projection operators onto the eigenvectors corresponding to $c_{i}$. If functions $f$ and $g$ satisfy $\hat{A}=f(\hat{C})$ and $\hat{B}=g(\hat{C})$, then $\hat{A}+\hat{B}$ can be written as

$$
\begin{aligned}
\hat{A}+\hat{B} & =f(\hat{C})+g(\hat{C}) \\
& =\sum_{i=1}^{N} f\left(c_{i}\right) \hat{P}_{i}^{C}+\sum_{i=1}^{N} g\left(c_{i}\right) \hat{P}_{i}^{C} \\
& =\sum_{i=1}^{N} h\left(c_{i}\right) \hat{P}_{i}^{C} \\
& =h(\hat{C}),
\end{aligned}
$$

where

$$
h\left(c_{i}\right) \equiv f\left(c_{i}\right)+g\left(c_{i}\right) .
$$

Therefore, the definite values of the left- and right-hand sides of the above equation also satisfy

$$
[A+B]=[h(C)] .
$$

On the other hand, the sum of $[A]$ and $[B]$ becomes

$$
\begin{aligned}
{[A]+[B] } & =[f(C)]+[g(C)] \\
& =f([C])+g([C]) \\
& =h([C]) \\
& =[h(C)] .
\end{aligned}
$$

Note that the second and fourth steps in Equation (95) are a consequence of FUNC. Thus, combining Equations (94) and (95) we obtain the sum rule

$$
[A+B]=[A]+[B] .
$$

The Kochen-Specker theorem can be proved using Equation (96). Let $|i\rangle, i=1, \cdots, M(M \leq N)$ be the orthonormal eigenstates of a given physical quantity, and

$$
\hat{P}_{i} \equiv|i\rangle\langle i|
$$

be the projection operator onto $|i\rangle$, which satsifies

$$
I=\sum_{i=1}^{M} P_{i}
$$

where $\hat{I}$ is the identity operator. By applying the sum rule (given by Equation 
(96)) on Equation (98), we obtain

$$
[I]=\sum_{i=1}^{M}\left[P_{i}\right] .
$$

Note that because all definite values belong to the spectrum of their corresponding operators, $[I]=1$ and $\left[P_{i}\right]=0$ or 1 . Thus, one $\left[P_{i}\right]$ must be 1 and the remaining 0 to satisfy Equation (99). In summary, if it is possible to assign every physical quantity a definite value satisfying FUNC, then one of the projection operators onto its eigenstate must be assigned a definite value 1 and the remaining must be assigned a definite value 0. Kochen and Specker [42] proved that it is not possible to assign such definite values to all physical quantities.

We reconsider the assumption FUNC. In FUNC, it is not possible to know which physical quantities are being observed simultaneously. Although it may seem natural that the definite values satisfy FUNC, Bell [43] insisted that contextual definite values do not need to satisfy FUNC. This implies that the Kochen-Specker theorem does not deny the existence of contextual definite values. We can say that the spin in each direction has a contextual definite value in the EPR-Bohm experiment introduced in Section 3.1, provided the definite value of the spin in the $r$ direction of particle 2 varies in accordance with the direction of the spin of particle 1, which was measured simultaneously. Furthermore, it is known that quantum mechanics must be nonlocal under such an assumption [44] [45].

\subsection{Nonlocality and Weak Values}

In view of the discussion in the previous sections, we need to check whether the counterfactual definiteness assumed during the derivations of the Bell-type inequalities is local. We consider the case in which the observed values are determined by local hidden variables, as shown in Equation (54). Note that Equation (58) is necessary for the derivation of $\mathrm{CHSH}$ inequality, and the density matrices for $A B$ and $A B$ ' must be the same to satisfy Equation (58). Therefore, we can conclude that the counterfactual definiteness assumed in this case is local. Thus, nonlocal counterfactual definiteness (i.e. existence of contextual definite values) is not forbidden in the above discussion. Conversely, if quantum mechanics is counterfactually indefinite, then we need to introduce nonlocality to explain the correlation between EPR pairs. Therefore, quantum mechanics must be nonlocal irrespective of whether it is counterfactually definite or indefinite, although it may seem difficult to appreciate this fact.

Weak measurements [46] clearly demonstrate the nonlocality of quantum mechanics. As confirmed by many experiments, the measured values in a weak measurement agree with the corresponding weak values. Nevertheless, the physical meaning of these concepts remained unclear for a long time. However, if we consider the nonlocality of quantum mechanics, then the physical meaning of weak measurements and weak values can be completely understood within the framework of a conventional quantum mechanical approach [47]. 


\section{Decoherence as an Inherent Characteristic of Quantum Mechanics}

As stated in the Introduction, most studies in this field have yielded a negative answer to the question regarding whether the measurement process can be explained within the framework of pure quantum mechanics. However, in this section, we demonstrate that the answer is affirmative, that is, we can indeed explain the measurement process within the framework of pure quantum mechanics. We propose a new decoherence theory, in which the uncertainty of microscopic objects gives rise to decoherence as an inherent characteristic of pure quantum mechanics. Note that because this decoherence exists prior to a measurement, the eigenstate-eigenvalue link can be maintained. Moreover, we do not intend to explain the nonlocality or counterfactual indefiniteness of quantum mechanics by means of other concepts, as it is beyond the scope of this work. However, we do attempt to illustrate how the measurement process can be understood within the scope of pure quantum mechanics.

We examine three experiments in this section. First, in Section 4.1, we examine a Stern-Gerlach-like experiment with an electron to illustrate our idea of decoherence. In Section 4.2, we apply our theory to an EPR pair of electrons and show that the correlation between spatially separated particles is not a result of wave packet collapse or similar processes. In Section 4.3, the double-slit experiment with electrons is examined to demonstrate the effectiveness of our theory for cases with continuous eigenvalues. This experiment also illuminates how pure quantum mechanics is able to demonstrate that electrons behave as interfering particles, that is, particles whose detection rate is consistent with interference.

\subsection{Stern-Gerlach-Like Experiment}

We examine a Stern-Gerlach-like experiment with an electron $S$ whose spin is measured in the $z$ direction, as shown in Figure 2. Note that $|+\rangle$ and $|-\rangle$ are the eigenstates of the electron with eigenvalues $+\hbar / 2$ and $-\hbar / 2$, respectively. $S$ initially travels along the $x$-axis and enters an inhomogeneous magnetic field along the $z$-axis, where a magnetic force acts on it. When $S$ exits the magnetic field, its momentum in the $z$ direction is $-p$ if its spin is $+\hbar / 2$ and $+p$ if the spin is $-\hbar / 2$. We define the momentum eigenstates $|-p\rangle$ and $|+p\rangle$ with eigenvalues $-p$ and $+p$, respectively. Furthermore, $|0\rangle$ is defined as the momentum eigenstate having 0 momentum. Note that because the spin and momentum operators along the same direction commute with each other, the state of $S$ can be described as a simultaneous eigenstate of these two operators. We also define a unitary operator $\hat{U}_{S}$ that represents the interaction between $S$ and the magnetic field, such that

$$
\begin{aligned}
& \hat{U}_{S}|+\rangle|0\rangle=|+\rangle|-p\rangle, \\
& \hat{U}_{S}|-\rangle|0\rangle=|-\rangle|+p\rangle .
\end{aligned}
$$




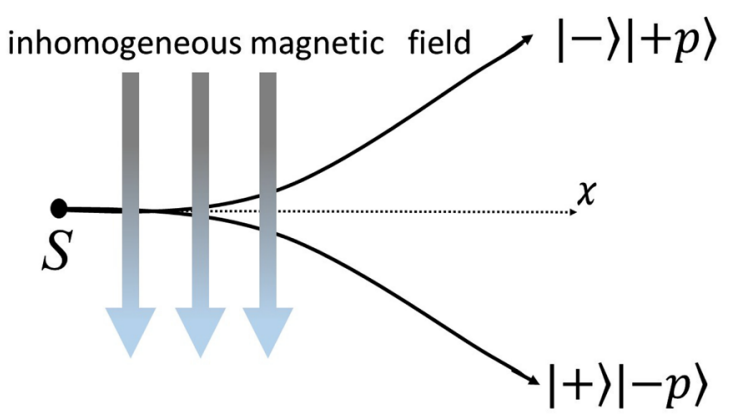

Figure 2. Stern-Gerlach-like experiment.

We define the initial state $\left|I_{S}\right\rangle$ of $S$ to be

$$
\left|I_{S}\right\rangle \equiv \frac{1}{\sqrt{2}}(|+\rangle+|-\rangle)|0\rangle \text {. }
$$

The state $\left|O_{S}\right\rangle$ after the interaction between $S$ and the magnetic field can be written as

$$
\left|O_{S}\right\rangle=\hat{U}_{S}\left|I_{S}\right\rangle=\frac{1}{\sqrt{2}}(|+\rangle|-p\rangle+|-\rangle|+p\rangle) \text {. }
$$

When $S$ reaches one of the detectors, the entire event is recorded. If we ignore the evolution of $S$ s state between the magnetic field and the detector, then $\left|O_{S}\right\rangle$ is the state of $S$ just before detection. The naïve density matrix $\hat{\rho}_{S 0}$ of $S$ is defined as

$$
\hat{\rho}_{S 0}=\left|O_{S}\right\rangle\left\langle O_{S}\right|
$$

Note that $\hat{\rho}_{S 0}$ and $\left|O_{S}\right\rangle$ differ in physical contents, as explained in the following.

Next, we consider the uncertainty relation. Because we want to measure $S$ s momentum in the $z$ direction, we must allow some uncertainty in its position in the same direction. To account for this uncertainty, we introduce the density matrix $\hat{\rho}_{S}(\zeta)$ of $S$ translated to a distance $\zeta$ in the $z$ direction, which is defined as

$$
\hat{\rho}_{S}(\zeta) \equiv \hat{T}_{z}(\zeta) \hat{\rho}_{S 0} \hat{T}_{z}^{\dagger}(\zeta)
$$

where $\hat{T}_{z}(\zeta)$ is the translation operator in the $z$ direction. $\hat{T}_{z}(\zeta)$ satisfies the following equation

$$
\left\langle z+\zeta\left|\hat{T}_{z}(\zeta)\right| O_{S}\right\rangle=\left\langle z \mid O_{S}\right\rangle
$$

and is defined by

$$
\hat{T}_{z}(\zeta) \equiv \exp \left(-\frac{i \hat{P}_{z} \zeta}{\hbar}\right)
$$

for the momentum operator $\hat{P}_{z}$ in the $z$ direction.

Next, we define the average density matrix $\hat{\rho}_{S a v}$ of $S$ as

$$
\hat{\rho}_{\text {Sav }} \equiv \frac{1}{2 \Delta z} \int_{-\Delta z}^{\Delta z} \mathrm{~d} \zeta \hat{\rho}_{S}(\zeta)
$$


where $2 \Delta z$ is the uncertainty in $S$ s position along the $z$ direction and from Equation (105) we can write

$$
\begin{aligned}
\hat{\rho}_{S}(\zeta)= & \frac{1}{2}\left(\exp \left(\frac{+i p \zeta}{\hbar}\right)|+\rangle|-p\rangle+\exp \left(\frac{-i p \zeta}{\hbar}\right)|-\rangle|+p\rangle\right) \\
& \times\left(\langle+p|\langle-| \exp \left(\frac{+i p \zeta}{\hbar}\right)+\langle-p|\langle+| \exp \left(\frac{-i p \zeta}{\hbar}\right)\right) .
\end{aligned}
$$

We want to know what is observed by the macroscopic detector; hence, we set

$$
\hbar \ll \Delta p \Delta z=2 p \Delta z,
$$

which leads to

$$
\frac{1}{2 \Delta z} \int_{-\Delta z}^{\Delta z} \mathrm{~d} \zeta \exp \left(\frac{ \pm 2 i p \zeta}{\hbar}\right) \simeq 0 .
$$

Therefore, the average density matrix, which describes the state of $S$ to be detected, loses its interference terms and becomes

$$
\hat{\rho}_{S a v}=\frac{1}{2}(|+\rangle|-p\rangle\langle-p|\langle+|+|-\rangle|+p\rangle\langle+p|\langle-|) .
$$

The above equation demonstrates the decoherence prior to the measurement process.

Note that $\hat{\rho}_{S a v}$ is not a consequence of the nonunitary (or unitary) time evolution from the state $\left|O_{S}\right\rangle$, but $\hat{\rho}_{\text {Sav }}$ is exactly the density matrix corresponding to $\left|O_{S}\right\rangle$ to be detected.

Note also that if condition (110) is weakened, then the interference terms in $\hat{\rho}_{S a v}$ would be retained to a certain extent. Performing the integral Equation in (108), we obtain

$$
\begin{aligned}
\hat{\rho}_{S a v}= & \frac{\hbar}{4 p \Delta z} \sin \left(\frac{2 p \Delta z}{\hbar}\right)(|+\rangle|-p\rangle+|-\rangle|+p\rangle)(\langle-p|\langle+|+\langle+p|\langle-|) \\
& +\frac{1}{2}\left(1-\frac{\hbar}{2 p \Delta z} \sin \left(\frac{2 p \Delta z}{\hbar}\right)\right)(|+\rangle|-p\rangle\langle-p|\langle+|+|-\rangle|+p\rangle\langle+p|\langle-|) .
\end{aligned}
$$

Because we do not adopt the projection postulate in this study, we observe in accordance with the eigenstate-eigenvalue link that the first term on the right-hand side of this equation does not contribute to the probability that $S$ will be detected as a particle with momentum $-p$ or $+p$ in the $z$ direction. Therefore, there is a $1-(\hbar / 2 p \Delta z) \sin (2 p \Delta z / \hbar)$ probability, which vanishes in the limit $\Delta z \rightarrow 0$, that $S$ will be detected as a particle with momentum $-p$ or $+p$ in the $z$ direction if we observe its position with an uncertainty $2 \Delta z$.

In studies that propose that the environment causes decoherence, an equation similar to Equation (112) is obtained by taking the partial trace of Equation (104). The state described by Equation (104) is a pure state; hence, the state described by the density matrix after the partial trace is an improper mixed state, which only provides the probability distribution of the outcome. By contrast, Equation (112) represents a proper mixed state and describes the state itself to be detected with the macroscopic detector. Therefore, we can conclude that the 
state to be detected is not given by Equation (103) but is either $|+\rangle|-p\rangle$ or $|-\rangle|+p\rangle$. In this calculation, we have not used any additional postulates such as the projection postulate. Thus, it is worth noting that this decoherence is not a result of the interaction between $S$ and the detector or other environmental factors, but is rather due to the uncertainty relation.

Note also that the density matrix of $S$ is not always written as shown in Equation (112). If we want to measure another observable of $S$, then we need to take an average the density matrix over its conjugate observables. Consequently, we can obtain the average density matrix that has a diagonal form in this observable, as illustrated in the remainder of this section.

Let us suppose that an ideal macroscopic measuring device $M$ that measures $S$ s energy is employed instead of the aforementioned detector. Furthermore, $S$ is prepared in its neutral state $|r\rangle$ and is at either of the two energy levels $E_{+}$ and $E_{-}$, in accordance with its spin, after it exits the magnetic field. $\hat{V}$ is a unitary operator that transforms $|r\rangle$ to $\left|E_{+}\right\rangle$or $\left|E_{-}\right\rangle$, which are the eigenstates whose eigenvalues are $E_{+}$and $E_{-}$, respectively. Thus, we have

$$
\begin{gathered}
\hat{V}|+\rangle|r\rangle=|+\rangle\left|E_{+}\right\rangle, \\
\hat{V}|-\rangle|r\rangle=|-\rangle\left|E_{-}\right\rangle .
\end{gathered}
$$

The initial state of the unified system is represented by $|J\rangle$, which is defined as

$$
|J\rangle \equiv \frac{1}{\sqrt{2}}(|+\rangle+|-\rangle)|r\rangle .
$$

Then, the state of $S$ just prior to detection by $M$ can be written as

$$
\hat{V}|J\rangle=\frac{1}{\sqrt{2}}\left(|+\rangle\left|E_{+}\right\rangle+|-\rangle\left|E_{-}\right\rangle\right),
$$

and its density matrix $\hat{\rho}_{J 0}$ is given by

$$
\hat{\rho}_{J 0}=\hat{V}|J\rangle\langle J| \hat{V}^{\dagger} .
$$

Note that because we want to measure the energy of $S$, we require a time interval. Therefore, we define the density matrix $\hat{\rho}_{J a v}$ averaged over the measurement time interval $2 \Delta t$ as

$$
\hat{\rho}_{J a v} \equiv \frac{1}{2 \Delta t} \int_{-\Delta t}^{\Delta t} \mathrm{~d} \tau \exp \left(-\frac{i \hat{H} \tau}{\hbar}\right) \hat{\rho}_{J 0} \exp \left(+\frac{i \hat{H} \tau}{\hbar}\right),
$$

where $\hat{H}$ is the Hamiltonian density of $S$, which satisfies

$$
\begin{aligned}
\hat{H}\left|E_{+}\right\rangle & =E_{+}\left|E_{+}\right\rangle, \\
\hat{H}\left|E_{-}\right\rangle & =E_{-}\left|E_{-}\right\rangle .
\end{aligned}
$$

Therefore, we can write

$$
\hat{\rho}_{J a v}=\frac{1}{2 \Delta t} \int_{-\Delta t}^{\Delta t} \mathrm{~d} \tau \hat{\rho}_{J}(\tau)
$$

with 


$$
\begin{aligned}
\hat{\rho}_{J}(\tau)= & \frac{1}{2}\left(\exp \left(\frac{-i E_{+} \tau}{\hbar}\right)|+\rangle\left|E_{+}\right\rangle+\exp \left(\frac{-i E_{-} \tau}{\hbar}\right)|-\rangle\left|E_{-}\right\rangle\right) \\
& \times\left(\left\langle E_{-}\right|\langle-| \exp \left(\frac{i E_{-} \tau}{\hbar}\right)+\left\langle E_{+}\right|\langle+| \exp \left(\frac{i E_{+} \tau}{\hbar}\right)\right) .
\end{aligned}
$$

To obtain a macroscopic result, we set

$$
\left(E_{+}-E_{-}\right) \Delta t \gg \hbar
$$

which leads to

$$
\frac{1}{2 \Delta t} \int_{-\Delta t}^{\Delta t} \mathrm{~d} \tau \exp \left(\frac{ \pm i\left(E_{+}-E_{-}\right) \tau}{\hbar}\right) \simeq 0 .
$$

Therefore, the average density matrix in this case becomes

$$
\hat{\rho}_{J a v}=\frac{1}{2}\left(|+\rangle\left|E_{+}\right\rangle\left\langle E_{+}|\langle+|+|-\rangle| E_{-}\right\rangle\left\langle E_{-}\right|\langle-|\right) .
$$

Thus, we observe that equation (126) is diagonal in $S$ s energy.

\subsection{EPR-Bohm Experiment}

In this section, we re-examine the EPR-Bohm experiment, which was briefly reviewed in Section 3.1. We adopt the same setup as in the previous section, where particles 1 and 2 enter inhomogeneous magnetic fields along the $z$ and $r$ directions, respectively. When the particles exit the magnetic fields, each particle gains a momentum in its respective direction of the magnetic field. Initially, neither particle has a momentum in the $z$ or $r$ direction. If we define the zero momentum state of the particles as $|0\rangle_{i}$, then the state $\left|I_{C}\right\rangle$ before entering the magnetic field can be written as

$$
\left|I_{C}\right\rangle=|C\rangle|0\rangle_{1}|0\rangle_{2},
$$

where $|C\rangle$ is defined in Equation (39). The state $\left|O_{C}\right\rangle$ after interaction between the electrons and magnetic fields becomes

$$
\begin{aligned}
\left|O_{C}\right\rangle= & \hat{U}_{C}\left|I_{C}\right\rangle \\
= & \frac{1}{\sqrt{2}}\left[|u\rangle_{1}|-p\rangle_{1}\left(\sin \theta|+\rangle_{2}|-q\rangle_{2}+\cos \theta|-\rangle_{2}|+q\rangle_{2}\right)\right. \\
& \left.-|d\rangle_{1}|+p\rangle_{1}\left(\cos \theta|+\rangle_{2}|-q\rangle_{2}-\sin \theta|-\rangle_{2}|+q\rangle_{2}\right)\right],
\end{aligned}
$$

where the unitary operator $\hat{U}_{C}$ represents the interaction, and $p$ and $q$ are the momenta in the $z$ and $r$ directions, respectively. As discussed in the previous section, we must allow some uncertainty in the position of the particles along the corresponding directions. Therefore, the density matrix $\hat{\rho}_{C a v}$ that describes the state to be measured is defined as

$$
\hat{\rho}_{C a v} \equiv \frac{1}{4 \Delta z \Delta r} \int_{-\Delta z}^{\Delta z} \mathrm{~d} \zeta \int_{-\Delta r}^{\Delta r} \mathrm{~d} \xi \hat{\rho}_{C}(\zeta, \xi)
$$

where $\hat{\rho}_{C}(\zeta, \xi)$ is the density matrix of particle 1 translated to a distance $\zeta$ in the $z$ direction and particle 2 translated to a distance $\xi$ in the $r$ direction. By using 


$$
\begin{aligned}
& \hat{T}_{1 z}(\zeta) \equiv \exp \left(-\frac{i \hat{P}_{1 z} \zeta}{\hbar}\right) \\
& \hat{T}_{2 r}(\xi) \equiv \exp \left(-\frac{i \hat{P}_{2 r} \xi}{\hbar}\right)
\end{aligned}
$$

where $\hat{P}_{1 z}$ and $\hat{P}_{2 r}$ are the momentum operators of particle 1 in the $z$ direction and particle 2 in the $r$ direction, respectively, and $\hat{\rho}_{C}(\zeta, \xi)$ can be written as

$$
\hat{\rho}_{C}(\zeta, \xi) \equiv \hat{T}_{1 z}(\zeta) \hat{T}_{2 r}(\xi)\left|O_{C}\right\rangle\left\langle O_{C}\right| \hat{T}_{2 r}^{\dagger}(\xi) \hat{T}_{1 z}^{\dagger}(\zeta) .
$$

Similar to the previous section, because we want to know what is observed by the macroscopic detectors, we set

$$
\hbar \ll p \Delta z, \hbar \ll q \Delta r,
$$

which leads to

$$
\begin{aligned}
\frac{1}{2 \Delta z} \int_{-\Delta z}^{\Delta z} \mathrm{~d} \zeta \exp \left(\frac{ \pm 2 i p \zeta}{\hbar}\right) & \simeq 0, \\
\frac{1}{2 \Delta r} \int_{-\Delta r}^{\Delta r} \mathrm{~d} \xi \exp \left(\frac{ \pm 2 i q \xi}{\hbar}\right) & \simeq 0 .
\end{aligned}
$$

Therefore, the interference terms disappear and the average density matrix takes a form similar to that in Equation (112):

$$
\begin{aligned}
\hat{\rho}_{C a v}= & \frac{1}{2}\left[\operatorname { s i n } ^ { 2 } \theta \left(| u \rangle | - p \rangle \left\langle-p|\langle u|)_{1}(|+\rangle|-q\rangle\langle-q|\langle+|)_{2}\right.\right.\right. \\
& +\cos ^{2} \theta\left(| u \rangle | - p \rangle \left\langle-p|\langle u|)_{1}(|-\rangle|+q\rangle\langle+q|\langle-|)_{2}\right.\right. \\
& +\cos ^{2} \theta\left(| d \rangle | + p \rangle \left\langle+p|\langle d|)_{1}(|+\rangle|-q\rangle\langle-q|\langle+|)_{2}\right.\right. \\
& +\sin ^{2} \theta\left(|d\rangle|+p\rangle\left\langle+p|\langle d|)_{1}(|-\rangle|+q\rangle\langle+q|\langle-|)_{2}\right] .\right.
\end{aligned}
$$

Because Equation (136) describes a proper mixed state, the state to be detected is not a superposition but rather one of the following states:

$(|u\rangle|-p\rangle)_{1}(|+\rangle|-q\rangle)_{2}, \quad(|u\rangle|-p\rangle)_{1}(|-\rangle|+q\rangle)_{2}, \quad(|d\rangle|+p\rangle)_{1}(|+\rangle|-q\rangle)_{2}, \quad$ and $(|d\rangle|+p\rangle)_{1}(|-\rangle|+q\rangle)_{2}$. It is worth noting that the correlation between the spins of the two particles should not be a result of the measurement process, as it is with a wave packet collapse, because Equation (136) represents the density matrix of the electrons prior to the measurement process. Therefore, we should not regard the EPR experiment as evidence for instantaneous propagation during a wave packet collapse.

Conversely, we need to consider the relation between the discussions in this and the previous section. Note that because we can obtain only one average density matrix for an observed pair of electrons, as discussed earlier, we do not need to assume that the state prior to the measurement possesses a definite value of the spin in each direction. In other words, the probable counterfactual definiteness is not local but contextual. As discussed in the previous section, contextual counterfactual definiteness is not forbidden by Bell-type inequalities or the Kochen-Specker theorem. The spin in either direction is not fixed in the initial state 
given by Equation (39), whereas the spin of each electron in the respective directions is fixed in state described by Equation (136). The transformation between Equations (39) and (136) is owing to the uncertainty relation. However, as stated in Section 1, we do not intend to explain the nonlocality of quantum mechanics by means of other concepts. We only attempt to illustrate how measurement processes can be understood within the scope of pure quantum mechanics in this study.

\subsection{Double-Slit Experiment}

In this section, we examine the double-slit experiment, where electrons are emitted from a source at certain time intervals (see Figure 3). The electrons travel along the $x$-axis through a double slit in the $x=0$ plane and eventually arrive somewhere on the screen in the $x=L$ plane. The slits are separated in the $z$ direction such that the two slits are positioned at $z=z_{1}$ and $z=z_{2}$. We define the eigenstate $|z\rangle$ of the $z$ coordinate operator $\hat{z}$ as

$$
\hat{z}|z\rangle=z|z\rangle \text {. }
$$

Moreover, we define $|\psi\rangle$ as the state of the electron at $x=X(0 \leq X<L)$, and $\left|\psi_{1}\right\rangle$ and $\left|\psi_{2}\right\rangle$ as the states obtained after the electron passes through slits 1 and 2, respectively. Thus,

$$
|\psi\rangle=\left|\psi_{1}\right\rangle+\left|\psi_{2}\right\rangle
$$

The wave function $\psi(z)$ is defined as

$$
|\psi\rangle=\int_{-\infty}^{\infty} \mathrm{d} z \psi(z)|z\rangle,
$$

with the normalisation

$$
\int_{-\infty}^{\infty} \mathrm{d} z|\psi(z)|^{2}=1
$$

The probability density that the electron is observed at $x=X, z=z_{0}$ is given by

$$
\begin{aligned}
\left\langle z_{0}\right\rangle & =\left\langle\psi \mid z_{0}\right\rangle\left\langle z_{0} \mid \psi\right\rangle \\
& =\int \mathrm{d} z \int \mathrm{d} z^{\prime} \psi^{*}\left(z^{\prime}\right) \psi(z)\left\langle z^{\prime} \mid z_{0}\right\rangle\left\langle z_{0} \mid z\right\rangle \\
& =\left|\psi\left(z_{0}\right)\right|^{2} .
\end{aligned}
$$

$\psi_{1}(z)$ and $\psi_{2}(z)$ can be defined in the same way as in Equation (139), which gives

$$
\begin{aligned}
& \left|\psi_{1}\right\rangle=\int_{-\infty}^{\infty} \mathrm{d} z \psi_{1}(z)|z\rangle, \\
& \left|\psi_{2}\right\rangle=\int_{-\infty}^{\infty} \mathrm{d} z \psi_{2}(z)|z\rangle .
\end{aligned}
$$

Then, we have

$$
\psi(z)=\psi_{1}(z)+\psi_{2}(z),
$$

and the probability density $\left|\psi\left(z_{0}\right)\right|^{2}$ is written as

$$
\left|\psi\left(z_{0}\right)\right|^{2}=\left|\psi_{1}\left(z_{0}\right)\right|^{2}+\left|\psi_{2}\left(z_{0}\right)\right|^{2}+2 \Re\left(\psi_{1}\left(z_{0}\right) \psi_{2}^{*}\left(z_{0}\right)\right) .
$$




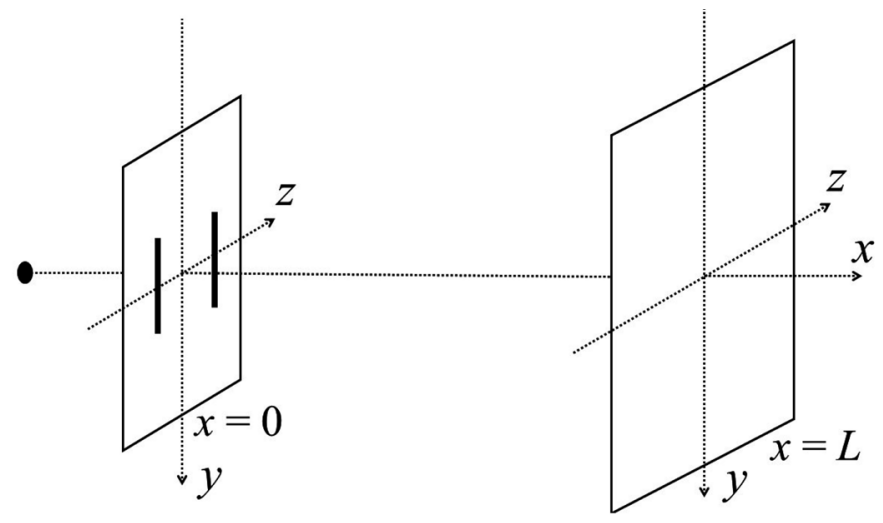

Figure 3. Double-slit experiment.

\subsubsection{Decoherence on the Screen}

In this section, the electrons are not assumed to be observed just after passing through the slits. Although the interference terms are included in the probability density $|\psi(z)|^{2}$, each electron behaves as a particle on the screen. Therefore, we illustrate here that the density matrix of the electrons to be observed on the screen is not given by $|\psi\rangle\langle\psi|$ but is rather proportional to

$$
\int \mathrm{d} z|\psi(z)|^{2}|z\rangle\langle z| \text {. }
$$

In contrast to the discussion in the previous sections, we need to allow some uncertainty in the momentum in the $z$ direction because we want to know the position in this direction. Therefore, we define the average density matrix $\hat{\rho}_{\psi a v}$ that describes the state of the electron to be observed on the screen as

$$
\hat{\rho}_{\psi a v} \equiv \frac{1}{2 h \Delta p} \int_{-\Delta p}^{\Delta p} \mathrm{~d} \pi \hat{\rho}_{\psi}(\pi),
$$

where $\hat{\rho}_{\psi}(\pi)$ is defined as

$$
\hat{\rho}_{\psi}(\pi) \equiv \hat{\mathcal{T}}(\pi)|\psi\rangle\langle\psi| \hat{\mathcal{T}}^{\dagger}(\pi),
$$

with

$$
\hat{\mathcal{T}}(\pi)=\exp \left(\frac{i \hat{z} \pi}{\hbar}\right) .
$$

$\hat{\mathcal{T}}(\pi)$ is the operator that transforms the momentum and satisfies

$$
\langle p+\pi|\hat{\mathcal{T}}(\pi)| \psi\rangle=\langle p \mid \psi\rangle,
$$

where $|p\rangle$ is the eigenstate of the momentum in the $z$ direction. Thus, we can write

$$
\hat{\rho}_{\psi a v}=\int_{-\Delta p}^{\Delta p} \frac{\mathrm{d} \pi}{2 h \Delta p} \int \mathrm{d} z \int \mathrm{d} z^{\prime} \psi^{*}\left(z^{\prime}\right) \psi(z) \exp \left(\frac{i\left(z-z^{\prime}\right) \pi}{\hbar}\right)|z\rangle\left\langle z^{\prime}\right| .
$$

In this experiment, we want to know where the electron impinges on the screen, therefore we set

$$
\hbar \ll\left(z-z^{\prime}\right) \Delta p
$$


which leads to

$$
\int_{\Delta p}^{\infty} \mathrm{d} \pi \exp \left(\frac{i\left(z-z^{\prime}\right) \pi}{\hbar}\right)=0
$$

Using the above equation, we can introduce a delta-function in Equation (151) as follows:

$\frac{1}{2 \Delta p} \int_{-\Delta p}^{\Delta p} \mathrm{~d} \pi \exp \left(\frac{i\left(z-z^{\prime}\right) \pi}{\hbar}\right) \sim \frac{1}{\delta(0)} \int_{-\infty}^{\infty} \mathrm{d} \pi \exp \left(\frac{i\left(z-z^{\prime}\right) \pi}{\hbar}\right)=\frac{h}{\delta(0)} \delta\left(z-z^{\prime}\right)$,

such that Equation (151) becomes

$$
\hat{\rho}_{\psi a v}=\frac{1}{\delta(0)} \int \mathrm{d} z \int \mathrm{d} z^{\prime} \psi^{*}\left(z^{\prime}\right) \psi(z) \delta\left(z-z^{\prime}\right)|z\rangle\left\langle\left. z^{\prime}\left|=\frac{1}{\delta(0)} \int \mathrm{d} z\right| \psi(z)\right|^{2} \mid z\right\rangle\langle z| .
$$

Note that Equation (155) is the desired form of the density matrix. The state that Equation (155) describes is a proper mixture, and hence, we can predict that each electron will behave as a particle. However, because $|\psi(z)|^{2}$ is a probability density that includes the interference terms, the electrons form a striped interference pattern when they impinge on the screen.

\subsubsection{Decoherence Near the Slits}

Next, we examine the case in which the electrons are assumed to be observed near either of the slits, namely, at $x=X \simeq 0$. In this case,

$$
\psi_{1}(z) \psi_{2}^{*}(z)=0,
$$

which follows from the definitions of $\psi_{1}(z)$ and $\psi_{2}(z)$. Therefore, equation (155) becomes

$$
\hat{\rho}_{\psi a v}=\frac{1}{\delta(0)} \int \mathrm{d} z\left(\left|\psi_{1}(z)\right|^{2}+\left|\psi_{2}(z)\right|^{2}\right)|z\rangle\langle z|,
$$

which shows that the interference terms have disappeared. If we now observe the electron on the screen again, its probability density is no longer given by $|\psi(z)|^{2}$ but rather by $\left|\psi_{1}(z)\right|^{2}+\left|\psi_{2}(z)\right|^{2}$.

\subsection{Summary}

We examined three fundamental experiments in this work. We studied the Stern-Gerlach-like experiment to explain how the uncertainty relation between the position and momentum of a particle introduces decoherence prior to a measurement. The EPR-Bohm experiment was considered to conclude that the correlation of the EPR pair is not a consequence of the instantaneous propagation during a wave function collapse. We also discussed its relation to the contextual counterfactual definiteness of quantum mechanics. Finally, we showed that our theory can be applied to experiments with continuous eigenvalues, such as the double-slit experiment with electrons. Thus, we demonstrated how pure quantum mechanics describes the fact that electrons behave as interfering particles, namely, particles whose detection rate is consistent with interference. 


\section{Conclusions}

In this study, we demonstrated that decoherence is one of the inherent characteristics of pure quantum mechanics. Quantum states describe both the wave as well as particle behaviour of microscopic objects, specifically, the microscopic objects propagate as waves and are observed as particles. In this context, it is worth paying careful attention to the meaning of the phrase observing the wave nature of a state. It is equivalent to saying identifying the state propagating as a wave by observing many particles. As shown in Section 4.3, there is essentially no difference between observing the electrons on the screen and near the slit. Irrespective of where the electrons are observed, they are observed as particles, which helps us determine the amplitude of the corresponding state.

Therefore, we conclude that the quantum measurement process can be explained within the framework of pure quantum mechanics. Moreover, we believe that our study can be applied to a more general discussion about the quantum-to-classical transition in nature.

\section{Conflicts of Interest}

The author declares no conflicts of interest regarding the publication of this paper.

\section{References}

[1] Schlosshauer, M., Kofler, J. and Zeilinger, A. (2013) Studies in History and Philosophy of Science Part B, 44, 222-230. https://doi.org/10.1016/j.shpsb.2013.04.004

[2] Laloë, F. (2013) Do We Really Understand Quantum Mechanics? Cambridge UP, Cambridge.

[3] Gilton, M.J.R. (2016) Studies in History and Philosophy of Science Part B: Studies in History and Philosophy of Modern Physics, 55, 92-100. https://doi.org/10.1016/j.shpsb.2016.08.005

[4] Schlosshauer, M. (2004) Reviews of Modern Physics, 76, 1267-1305. https://doi.org/10.1103/RevModPhys.76.1267

[5] Zurek, W.H. (2005) Physical Review A, 71, Article ID: 052105. https://doi.org/10.1103/PhysRevA.71.052105

[6] Born, M. (1926) Zeitschrift für Physik, 37, 863-867. https://doi.org/10.1007/BF01397477

[7] von Neumann, J. (1932) Mathematische Grundlagen der Quantenmechanik. Springer-Verlag, Berlin.

[8] Peres, A. and Terno, D.R. (2004) Reviews of Modern Physics, 76, 93. https://doi.org/10.1103/RevModPhys.76.93

[9] Everett, H. (1957) Reviews of Modern Physics, 29, 454. https://doi.org/10.1103/RevModPhys.29.454

[10] DeWitt, B.S. and Graham, N. (2016) The Many-Worlds Interpretation of Quantum Mechanics. Princeton UP, New Jersey.

[11] Joos, E., Zeh, H.D., Kiefer, C., Giulini, D., Kupsch, J. and Stamatescu, I.-O. (2003) Decoherence and the Appearance of a Classical World in Quantum Theory. 2nd Edition, Springer-Verlag, Berlin. https://doi.org/10.1007/978-3-662-05328-7 
[12] Schlosshauer, M. (2007) Decoherence and the Quantum-to-Classical Transition. Springer-Verlag, Berlin.

[13] Saunders, S., Barrett, J., Kent, A. and Wallace, D. (2010) Many Worlds? Everett Quantum Theory and Reality. Oxford UP, Oxford. https://doi.org/10.1093/acprof:oso/9780199560561.001.0001

[14] Zeh, H.D. (1970) Foundations of Physics, 1, 69-76. https://doi.org/10.1007/BF00708656

[15] Kübler, O. and Zeh, H.D. (1973) Annals of Physics, 76, 405-418. https://doi.org/10.1016/0003-4916(73)90040-7

[16] Zurek, W.H. (1981) Physical Review D, 24, 1516-1525. https://doi.org/10.1103/PhysRevD.24.1516

[17] Zurek, W.H. (1982) Physical Review D, 26, 1862-1880. https://doi.org/10.1103/PhysRevD.26.1862

[18] d'Espagnat, B. (1976) Conceptual Foundations of Quantum Mechanics. 2nd Edition, W. A. Benjamin, San Francisco.

[19] Dirac, P.A.M. (1958) The Principles of Quantum Mechanics. 4th Edition, Oxford UP, London.

[20] Landau, L.D. and Lifshitz, E.M. (1977) Quantum Mechanics (Non-Relativistic Theory). 3rd Edition, Pergamon Press, Oxford.

[21] Messiah, A. (1961) Quantum Mechanics. Wiley, New York.

[22] Blokhintsev, D.I. (1964) Quantum Mechanics. Springer, Berlin. https://doi.org/10.1007/978-94-010-9711-6

[23] Feynman, R.P. and Hibbs, A.R. (1965) Quantum Mechanics and Path Integrals. McGraw-Hill, New York.

[24] Schiff, L.I. (1968) Quantum Mechanics. 3rd Edition, McGraw-Hill, New York.

[25] Ehrenfest, P. (1927) Zeitschrift für Physik, 45, 455. https://doi.org/10.1007/BF01329203

[26] Freedman, S.J. and Clauser, J.F. (1972) Physical Review Letters, 28, 938-941. https://doi.org/10.1103/PhysRevLett.28.938

[27] Aspect, A., Grangier, P. and Roger, G. (1981) Physical Review Letters, 47, 460-463. https://doi.org/10.1103/PhysRevLett.47.460

[28] Aspect, A., Grangier, P. and Roger, G. (1982) Physical Review Letters, 49, 91-94. https://doi.org/10.1103/PhysRevLett.49.91

[29] Aspect, A., Dalibard, J. and Roger, G. (1982) Physical Review Letters, 49, 1804-1807. https://doi.org/10.1103/PhysRevLett.49.1804

[30] Ghosh, R. and Mandel, L. (1987) Physical Review Letters, 59, 1903-1905. https://doi.org/10.1103/PhysRevLett.59.1903

[31] Michler, M., Weinfurter, H. and Zukowski, M. (2000) Physical Review Letters, 84, 5457-5460. https://doi.org/10.1103/PhysRevLett.84.5457

[32] Hasegawa, Y., Loidl, R., Badurek, G., Baron, M. and Rauch, H. (2003) Nature, 425, 45-48. https://doi.org/10.1038/nature01881

[33] Einstein, A., Podolsky, B. and Rosen, N. (1935) Physical Review, 47, 777-780. https://doi.org/10.1103/PhysRev.47.777

[34] Bohm, D. (1951) Quantum Theory. Prentice-Hall, Englewood Cliffs.

[35] Clauser, J.F., Horne, M.A., Shimony, A. and Holt, R.A. (1969) Physical Review Letters, 23, 880-884. https://doi.org/10.1103/PhysRevLett.23.880 
[36] Clauser, J.F. and Horne, M.A. (1974) Physical Review D, 10, 526-535. https://doi.org/10.1103/PhysRevD.10.526

[37] Bell, J.S. (1964) Physics, 1, 195-200. https://doi.org/10.1103/PhysicsPhysiqueFizika.1.195

[38] Wigner, E.P. (1970) American Journal of Physics, 38, 1005. https://doi.org/10.1119/1.1976526

[39] Mermin, N.D. (1981) American Journal of Physics, 49, 940. https://doi.org/10.1119/1.12594

[40] Alberrt, D.Z. (1992) Quantum Mechanics and Experience. Harvard UP, Cambridge.

[41] Maccone, L. (2013) American Journal of Physics, 81, 854. https://doi.org/10.1119/1.4823600

[42] Kochen, S. and Specker, E. (1967) Journal of Mathematics and Mechanics, 17, 59. https://doi.org/10.1512/iumj.1968.17.17004

[43] Bell, J.S. (1966) Reviews of Modern Physics, 38, 447. https://doi.org/10.1103/RevModPhys.38.447

[44] Redhead, M. (1987) Incompleteness, Nonlocality, and Realism. Oxford UP, Oxford.

[45] Brown, H.R. and Svetlichny, G. (1990) Foundations of Physics, 20, 1379-1387. https://doi.org/10.1007/BF01883492

[46] Aharonov, Y., Albert, D.Z. and Vaidman, L. (1988) Physical Review Letters, 60, 1351. https://doi.org/10.1103/PhysRevLett.60.1351

[47] Mochizuki, R. (2018) International Journal of Theoretical Physics, 57, 1338. https://doi.org/10.1007/s10773-018-3662-1 